\title{
STUDIES ON THE VELOCITY OF BLOOD FLOW
}

VI. The Method of Collecting the Active Deposit of Radium and Its Preparation for Intravenous InJeCtion ${ }^{1}$

BY HERRMANN L. BLUMGART AND SOMA WEISS

(From the Thorndike Memorial Laboratory, Boston City Hospital and the Department of Medicine, Harvard Medical School)

(Received for publication April 13, 1927)

In establishing a satisfactory method for measuring the velocity of blood flow by means of the intravenous injections of radium $\mathrm{C}$, the collection of the active deposit of radium from radium emanation (radon) and the preparation of the active deposit for intravenous injection became problems of the utmost importance.

\section{A. THE METHOD OF COLLECTING THE ACTIVE DEPOSIT OF RADIUM}

The problem. Radium emanation is, of course, a gas. A consideration of the problem involved showed that a satisfactory means of collecting the active deposit of radium from radium emanation would have to fulfill the following requirements: (a) The method must provide some means of combining the contents of a number of tubes each containing a relatively small amount of radium emanation. The reason for this is a practical one. The tubes containing 20 or more millicuries of radium emanation are valuable for therapeutic purposes, whereas tubes containing $\mathbf{5}$ or $\mathbf{1 0}$ millicuries are more limited in their usefulness and can therefore be dispensed with at times. In order to utilize these tubes of emanation it was therefore essential to devise some means whereby, for example, five tubes containing 10 millicuries each could be crushed and the resulting 50 millicuries utilized in one unit as a source of active deposit of radium. (b) The process of crushing the tubes and the transfer of the liberated emanation to a proper receptacle must be accomplished without the possi-

1 This investigation was aided by a grant from the Proctor Fund of the Harvard Medical School for the Study of Chronic Diseases. 
bility of loss of any radium emanation. The escape of even a minute amount might result in the diffusion of the gas about the laboratory. Since the method of measuring the velocity of blood flow depends upon a detecting device of great sensitivity, liberated emanation might disturb the entire experimental procedure. A loss of radium emanation would, moreover, not be economical. (c) The chamber for holding the emanation must be absolutely air tight but must nevertheless permit the introduction and withdrawal of the electrode used for collecting the active deposit without allowing any of the emanation to escape. (d) The amount of active deposit collected must be a reasonably high percentage of that theoretically possible. Unless this requirement were fulfilled, the amount of radium emanation necessary would be unduly large. (e) The procedure must use the same radium emanation for repeated collections of active deposit without the loss of any emanation save through the natural rate of its decay. ( $f$ ) The active deposit of radium must be collected without any possible contamination by radium emanation or by any other substance which might be deleterious if injected intravenously. (g) The entire procedure must be simple and dependable.

The procedure adopted. Initial attempts were made to collect the active deposit upon sodium chloride which was exposed to the radium emanation in a high vacuum. The procedure was found to be uncertain and time-consuming. It therefore seemed that a simpler method which did not necessitate high vacua might prove more reliable.

Rutherford (1) showed in 1900 that when an emanating compound of thorium is placed in a positively charged, closed vessel, the active deposit could be concentrated on a negatively charged wire. H. W. Schmidt (2), and Wellisch and Bronson (3), in 1908 and 1912 respectively, demonstrated that, similarly, the active deposit of radium could be concentrated on the negative electrode if the emanation were exposed to strong electric fields. These investigators showed that, in a field of about 200 volts, between 80 and 90 per cent of the active deposit could be concentrated on the insulated, axially situated negative electrode. The conditions of their experiments hardly conformed to the requirements of the collection of the active deposit for intravenous injection. Though these investigators did not state 
the actual quantity of active deposit collected, they were presumably dealing with relatively small amounts of radium emanation where high yields would be more easily attainable.

After considerable experimentation we have found the design of

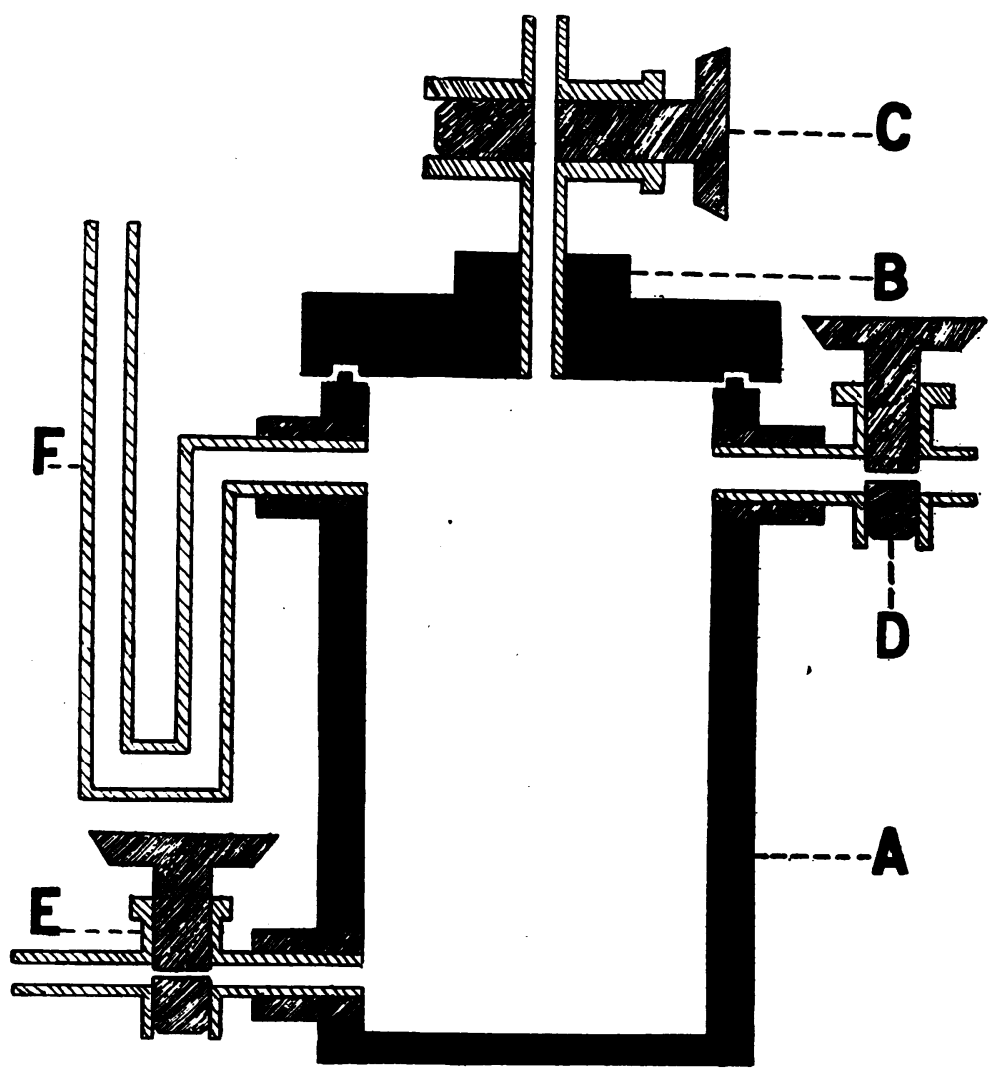

Fig. 1. Diagram of Emanation Chamber Used for the Collection of the Active Deposit of Radium

the chamber figured below to be adequate for the preparation of the active deposit of radium. The steel chamber (fig. 1) which contains the radium emanation consists of the cylinder $A$, to which is fitted a hard rubber top plate $B$. The steel chamber $A$ is $9 \mathrm{~cm}$. high and $5 \mathrm{~cm}$. in diameter. Stopcock $C$ whose bore is centrally situated in 
respect to the cylinder allows one to introduce the platinum electrode upon which the active deposit is to be collected. Stopcock $E$ permits the introduction into or escape of mercury from the chamber. A glass manometer tube is inserted at $F$ in order that one can observe at all times the relation of the pressure within the chamber to the pressure existing in the room. The radium emanation is introduced into the chamber through stopcock $D$. All stopcocks are accurately fitted to the chamber and then sealed by means of DeKhotinsky cement. The rubber top is sealed to the steel chamber by means of heavy stopcock grease.

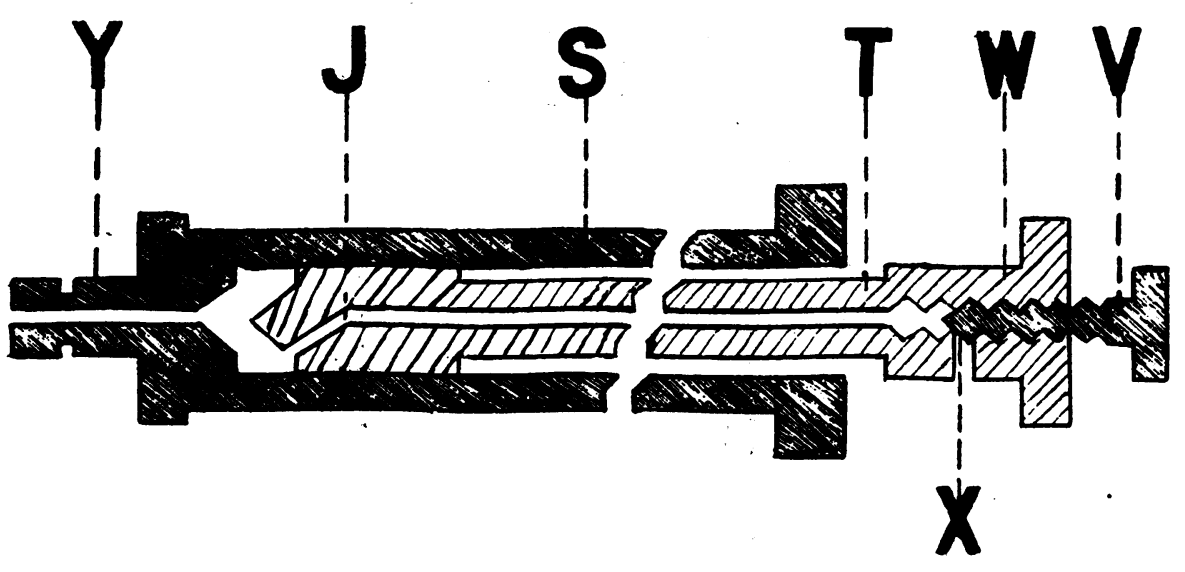

Fig. 2. Diagram of Device Used for Crushing Glass Tubes Containing Radium Emanation and Introducing Liberated Emanation iNTO CHAMBer

The radium emanation tubes are crushed by means of the breaking device shown in figure 2. $S$ is the barrel of an ordinary $5 \mathrm{cc}$. "Record Syringe". The handle usually attached to the plunger was removed and in its stead a hollow tube was soldered to the piston $J$. The bore of the hollow brass tube $T$ communicates at one end through a small hole drilled in the piston with the interior of the barrel of the syringe, and at the other end through a needle valve arrangement $V X W$ with the room air.

The actual procedure is as follows. The walls of the steel emanation chamber are carefully cleaned and mercury is introduced into the 
chamber so that the level of the mercury is $5 \mathrm{~cm}$. below the top of the chamber. The manometer $F$ is filled with mercury and the top rubber plate $B$ is sealed on. Stopcock $D$ is closed and negative pressure is established in the chamber by aspirating air through stopcock $C$. The chamber is tested for several hours to be certain that it is air tight.

The breaking device is then attached to stopcock $D$ of the emanation chamber by means of a short rubber connection. Next the small glass tubes containing the radium emanation are placed at the bottom of the syringe and the plunger is inserted into the barrel. By keeping the needle valve freely open the tip of the plunger can be

\begin{tabular}{|c|c|c|c|c|}
\hline $\begin{array}{l}\text { Determination } \\
\text { number }\end{array}$ & $\begin{array}{l}\text { Duration } \\
\text { activation }\end{array}$ & $\begin{array}{l}\text { Millicuries of } \\
\text { radon in source }\end{array}$ & $\begin{array}{l}\text { Millicuries on } \\
\text { electrode }\end{array}$ & $\begin{array}{l}\text { Percentage } \\
\text { of the theoretical } \\
\text { obtainable }\end{array}$ \\
\hline & minutes & millicuries & millicuries & per cent \\
\hline 1 & 60 & 39 & 10.7 & 54 \\
\hline 2 & 65 & 34 & 12.2 & 64 \\
\hline 3 & 90 & 26 & 11.2 & 58 \\
\hline 4 & 180 & 25 & 13.4 & 54 \\
\hline 5 & 240 & 17 & 8.9 & 52 \\
\hline 6 & 60 & 53 & 16.5 & 60 \\
\hline 7 & 50 & 53 & 11.9 & 54 \\
\hline 8 & 50 & 53 & 14.7 & 67 \\
\hline 9 & 110 & 45 & 18.8 & 48 \\
\hline 10 & 50 & 51 & 14.6 & 67 \\
\hline
\end{tabular}

brought down close to the emanation tubes. The needle valve is then screwed shut. A small piece of rubber tubing is attached to the manometer $F$ and clamped with a hemostat. Stopcock $C$ is then connected to a source of high vacuum and the chamber is exhausted with all other stopcocks closed. Stopcock $C$ is then closed, and stopcock $D$ is slowly opened. The tubes in the syringe are then crushed by the plunger $J$. By opening the needle valve $V$, (fig. 2) to the room air at $X$ and at the same time rotating the plunger $T W$, the liberated radium emanation is washed into the chamber which is now at negative pressure. The radium emanation being now delivered to chamber $A$, stopcock $D$ is closed. The emanation being now safe in the chamber $A$, room pressure is again established either 
by allowing mercury to draw through stopcock $E$ or by allowing air to enter through $C$ until the manometer pressure reading is zero. To arrange for this new (room) pressure is important, for the opening $C$, through which the platinum needle is now to be introduced, could not be sealed in order to retain the emanation by a few drops of mercury unless inside and outside pressures were equal. A centi-
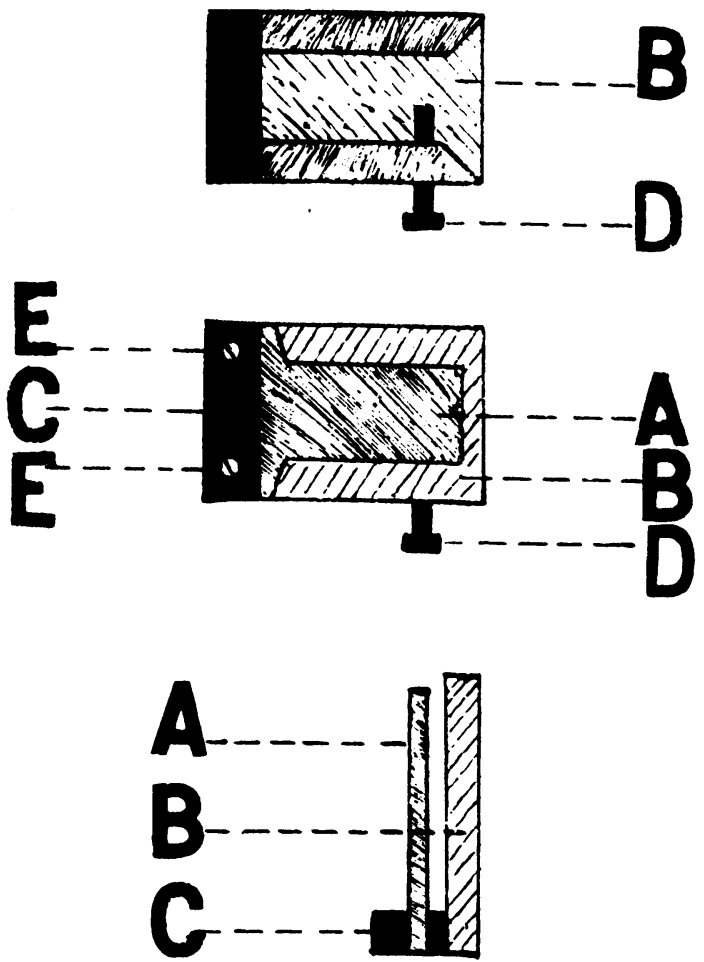

Fig. 3. Diagram of Clip for Automatically Recording Time of Injection

meter length of rubber tubing is accordingly attached to stopcock $C$ and filled with a few drops of mercury. A length of No. 20 platinum wire on which, as has been said active deposit is to be collected is inserted through stopcock $C$ into the chamber. It is important that the point of the platinum needle be $2.5 \mathrm{~cm}$. above the level of the mercury. The tip of the wire should be sharp. The wire is 


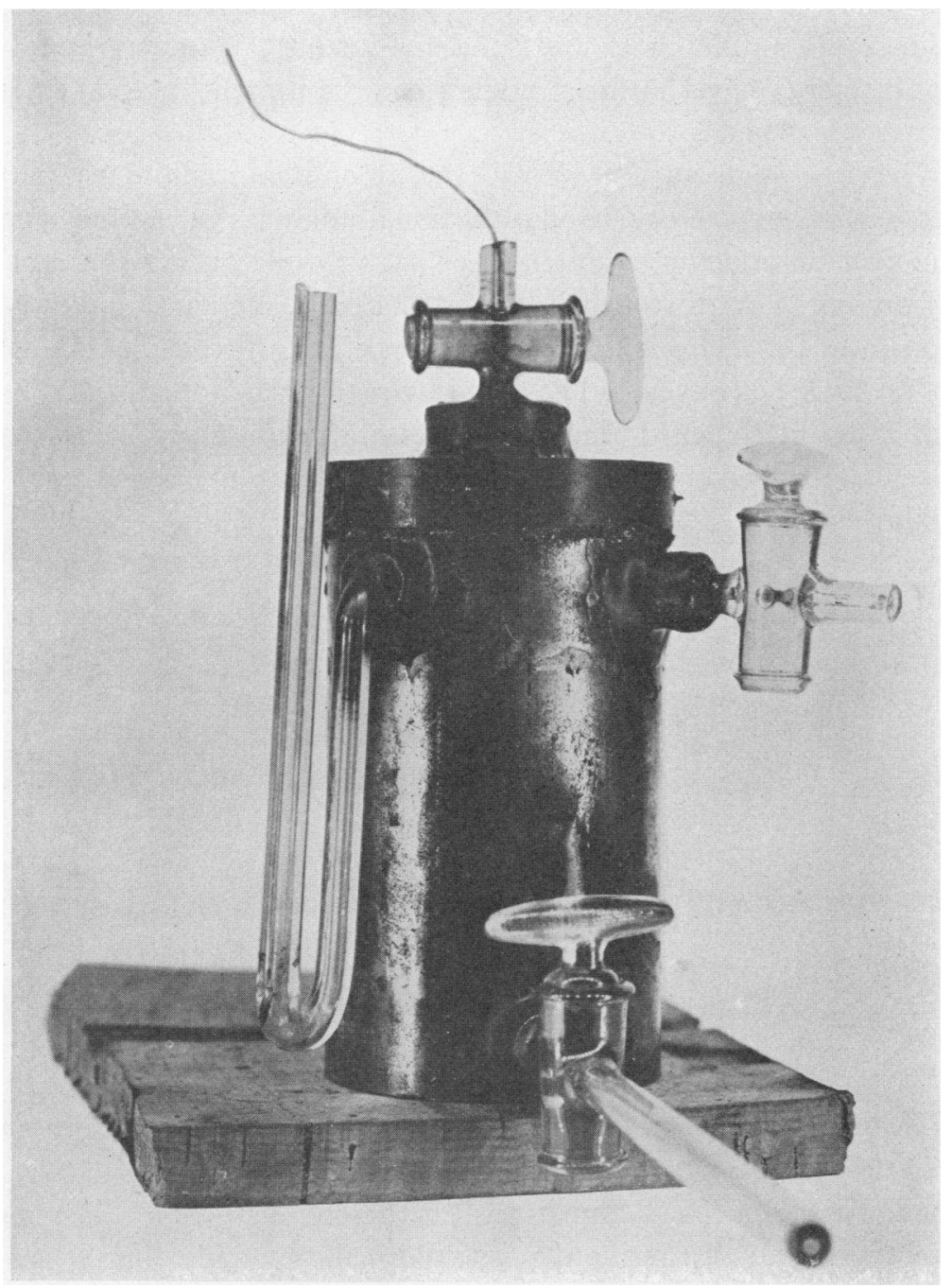

Fig. 4. Photograph of Emanation Chamber Used for the Collection of Active Deposit of Radium

connected to the negative pole of a 220 -volt line while the steel chamber is connected to the positive pole. By altering the dimensions it is probable that 110 volts could be used. 
The results obtained by the procedure are given in table 1 . Higher yields could probably be obtained by further experimentation but such attempts have not been made since the present method satisfies our practical needs.

When, through natural decay, the amount of radium emanation becomes inconveniently small, additional amounts of radium emanation may be added to the chamber without discarding the amount still present. With stopcocks $C$ and $D$ closed, mercury is allowed to drain from the chamber through stopcock $E$ until considerable negative pressure has developed as noted by reading manometer $F$. Stopcock $D$ is then opened, the new tubes are crushed according to the procedure previously outlined, the needle valve is opened, and the radium emanation washed into the chamber.

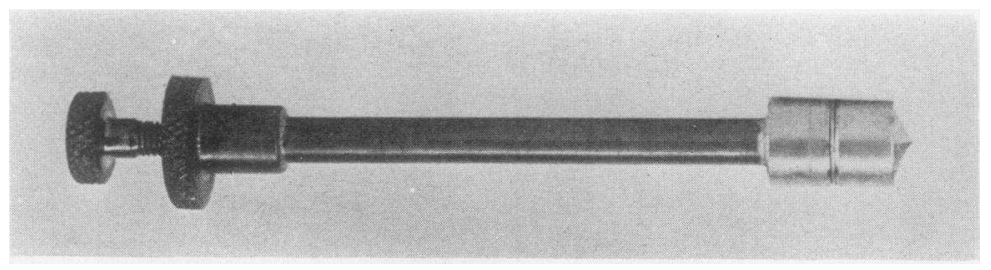

Fig. 5. Device Used for Crushing Emanation Tubes and Introducing the Radium Emanation into Chamber

B. THE METHOD OF PREPARATION OF THE ACTIVE DEPOSIT AND ITS INTRAVENOUS INJECTION

The platinum needle is removed from $\mathrm{C}$ and inserted into a small glass tube which has previously been half filled with 10 per cent hydrochloric acid. The active deposit is thereby dissolved. The needle is removed and the active deposit hydrochloric acid solution is neutralized to phenol red by means of $\mathrm{NaOH}$. The volume of the resultant solution is not more than $0.1 \mathrm{cc}$. The dissolved active deposit $\mathrm{NaCl}$ solution is drawn up into a tuberculin syringe and is then ready for use. The syringe is attached to a three-way stopcock to one opening of which a needle is attached for intravenous injection. The other connection communicates with a manometer containing 
citrate solution, by means of which the venous pressure is measured according to the method of Moritz and Tabora (4).

To the handle of the plunger of the syringe is attached a small clip (fig. 3). $B$ is a brass shoulder which fits over the flange of the syringe handle and is firmiy secured by the screw $D . A$ is a thin leaf of copper which is inserted into the hard rubber $C$ and insulated by it from $B$. When the intravenous injection is accomplished by pressure on the leaf $A$, an electrical circuit is closed through $A$ and $B$ and the time and duration of the intravenous injection are automatically recorded on the moving tape of a siphon feed galvanometer recorder.

We wish to thank Dr. William Duane for his interest and advice.

\section{CONCLUSIONS}

1. The details of a method for collecting the active deposit of radium from radium emanation and its preparation for intravenous injection are described.

2. Because of the simplicity of the procedure and the relatively high yjeld of active deposit attainable, the method is well suited to the purposes of the determination of the velocity of blood flow in man.

3. When, through the natural period of decay, the amount of radium emanation becomes inconveniently small, additional amounts of radium emanation can be added to the chamber without discarding the amount in previous use.

4. The details of a method for breaking several tubes each containing a small amount of radium emanation, and the process of transferring the liberated gas to an ionization chamber without loss of any of the emanation is described.

5. A device for automatic registration of the time and duration of intravenous injection is described.

\section{BIBLIOGRAPHY}

1. Rutherford, E., London, Edinburgh, and Dublin Philosophical Magazine and Journal of Science, 1900, 5 s, xlix, 161. Radioactivity Produced in Substances by the Action of Thorium Compounds.

2. Schmidt, H. W., Physikalische Zeitschrift, 1908, ix, 184. Ueber die Aktivierung der zentralen Elektrode eines zylindrischen Gefäsze in Radium Emanation. 
3. Wellisch, E. M., and Bronson, H. L., London, Edinburgh, and Dublin Philosophical Magazine and Journal of Science, 1912, $6 \mathrm{~s}$, xxiii, 714. The Distribution of the Active Deposit of Radium in an Electric Field.

4. Moritz, F., and Tabora, D. V., Deutsch. Arch. f. klin. med., 1910, xcviii, 475. Ueber eine Methode, beim Menschen den Druck in Oberflächlichen Venen exakt zu bestimmen. 